Témoigner Témoigner. Entre histoire et mémoire

Getuigen Revue pluridisciplinaire de la Fondation Auschwitz

$126 \mid 2018$

Questions sur l'avenir du travail de mémoire

\title{
Les étapes de la discussion sur la Shoah dans la société israélienne
}

The Evolution of Holocaust Remembrance in Israeli Society

\section{Gideon Greif}

Traducteur : Anna Gorbatova

\section{(2) OpenEdition \\ Journals}

\section{Édition électronique}

URL : https://journals.openedition.org/temoigner/7195

DOI : $10.4000 /$ temoigner.7195

ISSN : 2506-6390

Traduction(s) :

The Evolution of Holocaust Remembrance in Israeli Society - URL : https://journals.openedition.org/ temoigner/7237 [en]

\section{Éditeur :}

Éditions du Centre d'études et de documentation Mémoire d'Auschwitz, Éditions Kimé

\section{Édition imprimée}

Date de publication : 2 avril 2018

Pagination : 68-78

ISBN : 978-2-930953-06-9

ISSN : 2031-4183

Référence électronique

Gideon Greif, «Les étapes de la discussion sur la Shoah dans la société israélienne », Témoigner. Entre histoire et mémoire [En ligne], 126 | 2018, mis en ligne le 20 janvier 2022, consulté le 04 février 2022. URL : http://journals.openedition.org/temoigner/7195; DOI : https://doi.org/10.4000/temoigner.7195 


\section{Les étapes de la discussion sur la Shoah dans la société israélienne}

$\rightarrow$ Gideon Greif

Lhistorien et pédagogue
israélien Gideon Greif
mene des recherches
au Shem Olam
Faith \& Holocaust
Institute for Education,
Documentation \&
Research, en Isräl, et à la
Foundation for Holocaust
Education Projects
à Miami, en Floride.
Son thème central de
recherche est le camp
d'Auschwitz et 'histoire
du Sonderkommando. II
a publei de nombreux
articles et tient des
conférences dans le
monde entier.

monde entier.

\section{INTRODUCTION}

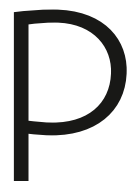

ur les détenus juifs qui ont vécu le jour de la Libération dans les camp e concentration et les centres d'extermination, la Shoah ne prit pas fin vec la capitulation de l'Allemagne national-socialiste. Seuls, empreint de douleur et de honte, ils tentèrent de commencer un nouveau chapitre de leur vie.

Quant à la population juive en Eretz Israel ${ }^{l}$ (dénommée Yichouv), elle était 列 due de l'horreur qui avait sévi en Europe, ayant observé le tout de très loin. Ce n'est que vers la fin de la guerre quelle commença à avoir de la compassion pour les victimes des crimes perpétrés par les nazis. Très vite, des reproches s'élevèrent parce que l'on n'avait pas suffisamment agi en faveur des personnes assassinées en Europe. La population juive de Palestine sous mandat britannique n'eut cependan pas beaucoup de temps à accorder au passé, car dès les années 1940, elle fut ellemême confrontée à des questions existentielles. Bien des années après la Shoah, les relations avec les survivants étaient distantes, et les malentendus et autres conflits dominaient le dialogue.

Cette distance fut renforcée par le fait que les survivants qui avaient échappé à l'enfer et immigré en Israël se retrouvèrent presque immédiatement confrontés à une nouvelle situation de guerre. Certains arrivèrent même pendant la guerre israélo-arabe de 1948 et durent prendre part aux combats, sans avoir jamais tenu d'arme à la main. Beaucoup furent tués ou gravement blessés, souvent parce qu'ils ne comprenaient pas la langue - l'hébreu - dans laquelle étaient donnés les ordres. On ne peut s'imaginer aujourd'hui ce que signifiait pour ces personnes, fortement traumatisées de devoir s'engager aussitôt dans une nouvelle guerre.

LA FAÇON D'ABORDER LA SHOAH ET SON IMPORTANCE POUR LA SOCIÉTÉ ISRAÉLIENNE

Une thèse centrale que nous souhaitons aborder ici stipule que, durant les vingt premières années après la fin de la Seconde Guerre mondiale, il n’y eut guère de débat public sur la Shoah en Israël. Quand on consulte les livres d'histoire et les ouvres de fiction, on a l'impression que le sujet été mis sous le boisseau pendant les années 1940 et $1950^{2}$.

Cette thèse soutient que le Premier ministre d'Israël, David Ben Gourion, fut l'un des principaux responsables de l'absence de prise en compte de la période nazie ; lui non plus n’aurait, dit-on, (Porat 2008b, p. 1lsqq) Lapresseisrálienne décrivaitjusquedans les années 1970 et 1980, la rencontre historique entre les Israéliens établis et les survivants immigrés comme antagoniste et caractérisait cette époque comme «le temps du silence » (Segev, 2003).

Si la représentation de la Shoah - en tant que sujet insignifiant et tabou jusquà ce qu'il fût thématisé «la Wende » (le tournant) - s'est développè dans un contexte de formation d'une nouvelle identité israélienne après la création de l'État, une recherche approfondie montre que cette représentation n'est que partiellement exacte. En fait, la Shoah a toujours accompagné et marqué la société israélienne depuis la période précédant les jours de la création de l'État israélien. et ont des répercussions déterminantes sur la société isrálienne et ce jusqu’à nos jours La Sho ho jours. La Shoah s'mmisçait constanment dans différents débats et discussions et a suscité un grand émi dé la ce qui a changé à bien des égards est la manière de se souvenir.

Parmi les premiers survivants qui immigrèrent en Eretz Israel après la fin de la Seconde Guerre mondiale, figuraient souvent des chefs de mouvements de résistance. Ils avaient organiséles combats dans des ghettos et dans des forêts. Parmi eux Ruzha Korczak et Abba Kowner de Vilnius ou Zivia Lubetkin et Yitzhak Zuckerman du ghetto de Varsovie (Porat, 2008b, p. 114sqq). Ils sillonnaient le pays comme des héros, racontaient leur histoire et publièrent une série de livres dans les années $1950^{3}$. Les racontain (a) plus importants de la Shoah. Ces actes étaient associés aux valeurs qui,jadis, étaient considérées comme particulièrement importantes en Israël : la défense nationale, la lutte pour la liberté et le développement du pays ${ }^{4}$.

En même temps, une attitude hostile s'est manifestée à l'égard d'une grande partie des Juifs d'Europe qui n'avaient pas luttés contre les nazis et qui, selon les avis désapprobateurs, auraient fait preuve de passivité, de faiblesse et de lâcheté. On disait même que les Juifs d'Europe auraient marché « comme des moutons allant à l'abattoir » (Bauer, 1989, p. 217sqq). Des attributs tels que la lâcheté ou la faiblesse étaient à l'époque en contradiction avec les valeurs fondamentales de la société israélienne. Ce comportement dédaigneux à l'égard des survivants s'appuyait en israélienne. Ce comportement dédaigneux a l'égard des survivants s'appuyait en
partie aussi sur les sentiments de culpabilité d'une partie de la société israélienne montré la moindre sensibilité à légard du sort des Juifs d'Europe Son souvenir et ses conséquences directes reviennent sans cesse dans la vie publique Shoah constitua un élément important du mythe fondateur de l'Etat d'Israël. Mais et des membres du FPO (Fareynikte Partizaner
Organizatsye, Organisatio unifiée des partisans) 
qui n’avait pas pu secourir ses frères et sours durant la Shoah. Celle-ci fut généralement interprétée comme un échec, auquel la société israélienne ne voulait pas prendre part ${ }^{5}$. Cette attitude fut essentiellement associée au renouveau et à la force retrouvée que l’on put voir dans la création de l'État d'Israël.

Outre l'attitude critique envers les survivants, des attaques violentes et des reproches sévères furent lancés à l'égard des conseils juifs (Judenräte) et d’autres organisations juives qui avaient existé durant la domination nazie. Ces critiques organisations juives qui avaient existé durant a donintion nazie. Ces critique cessèrent seulement à la fin des années 1980 ou furent remplacées progressivement par une attitude plus nuancée. Pendant longtemps cependant, on n’arrivait pas à distinguer les zones grises, car on ne parvenait pas à comprendre dans quelles conditions et sous quelles pressions les Juifs d'Europe avaient dû prendre des décisions morales parfois importantes ${ }^{6}$.

La longue guerre israélo-arabe renforça cette tendance, avec elle se répandit la reconnaissance de la lutte armée et la critique stéréotypée d’une prétendue « Diaspora de Juifs passifs » (Segev, 2003) s’intensifia. Les récits négatifs sur les Juifs parqués dans des camps de personnes déplacées en Europe (DP, Displaced Persons), que les Alliés avaient érigés pour les survivants dans bien des cas sur le territoire (Ditore allemand, accentuèrent le côté dévalorisant. Ils véhiculèrent une image négative de Juifs d'Europe, en mentionnant qu'ils s'adonnaient au marché noir, qu'ils étaient peu autonomes et improductifs. Suivant ces préjugés, on partait de l'idée que les survivants dans les camps de déplacés n’étaient pas honnêtes et vertueux parce que l'on supposait en Israël que - à l'exception de quelques rares héros que l’on célébrait - seuls des gens corrompus, égoïstes et vils avaient pu survivre au massacre tandis que les honnêtes gens étaient décédés.

La grande vague des survivants de la Shoah a immigré en Israël au cours des Lnées 1948 et 1949. Ils se heurtèrent à un modèle social dominant qui condamnait la faiblesse, la passivité et la lâcheté Des traits de caractère dont on dit qu'ils

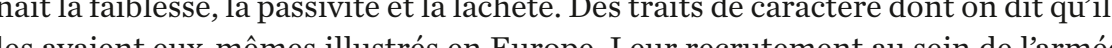
les avaient eux-mêmes illustrés en Europe. Leur recrutement au sein de l'armée était dès lors particulièrement compliqué - pour les survivants ce fut une épreuve quotidienne. D'un côté, ils voulaient prouver leur esprit de résistance, de l'autre, nombre d'entre eux étaient les seuls survivants de leur famille. Ce fut d'autant plus douloureux qu'ils furent nombreux à tomber au cours des combats: environ un tiers des soldats de l’armée israélienne participant à la guerre israélo-arabe étaient des survivants de la Shoah (Yablonka, 1999, p. 82;1995, p. 557sqq). Le combat commun avec les autochtones contribua au moins partiellement à acquérir le sentiment d’appartenir au pays d'Israël et à compenser les événements souvent traumatisants de la perte de leur famille et de leur patrie. De nombreux d'entre eux purent désormais affronter l'avenir avec un courage et une force renouvelés et le nourel État fut du moins en partie a la reconstruction. Ils considérèrent chaque nouveau chapitre de leur vie comme une victoire sur les nazis qui auraient voulu rendre le monde entier « judenrein ». («nettoyer » le monde entier des Juifs).

Dans les années 1950, les activités, les projets et les discussions publiques ayant un lien avec la Shoah se multiplièrent. Ils furent initiés et dirigés par diverses organisations de survivants. Ceux-ci exercèrent alors davantage de pression sur le gou-

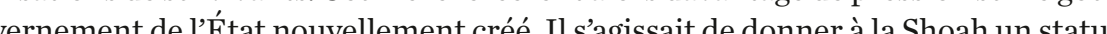
Cex-ciextut «officiel» ainsi qu un role central dans la conscience publique afin que les survivants se sentent membres à part entière de la nouvelle société. Avec cette reconnaissance publique, les rescapés ne vivaient plus seulement en marge de la société, mais intervenaient efficacement tout en gagnant toujours plus de poids politique et d'influence. Au sein du Parlement israélien siégèrent aussi un certain nombre de survivants. Leur engagement pour institutionnaliser la mémoire de la Shoah aboutit à l'adoption de quelques lois : Le 9 août 1950, la première version de la « loi sur la condamnation des nazis et de leurs collaborateurs entra en vigueur. $»^{8}$ Elle permit aux tribunaux israéliens de condamner plus tard Eichmann et Demjanjuk pour les crimes perpétrés pendant la Shoah. Le 28 août 1953 fut adoptée la « loi sur la mémoire de la Shoah et de l'héroïsme». Dans le cadre de de l'héroïsme ». Dans le cadre de celle-ci fut aussi créé le mémorial israélien officiel
consacré à la Shoah : Yad Vashem?.

En 1954 fut promulguée par ailleurs la «loi pour l’assistance aux blessés de guerre et aux invalides », qui fut suivie le 8 avril 1959 par la « loi sur la journée de la mémoire de la Shoah et de l'héroïsme ».

Les années 1950 furent marquées par un conflit intense polarisant fortement la société israélienne. En 1952, commença la discussion sur le postulat des dites «Indemnisations des victimes de la Shoah » de la République fédérale d’Allemagne. Cette discussion allait insuffler à la Shoah une nouvelle dimension dans le discours politique.

En arrière-plan, le contexte était le suivant : Le Premier ministre Ben Gourion avait essayé de trouver un accord avec la République fédérale d’Allemagne afin de renforcer l'économie de son pays, encore en cours de construction. Des milieux influents forcer l'économie de son pays, encore en cours de construction. Des milieux influents
s'opposèrent avec véhémence à tout rapprochement de peur d'ouvrir la voie à une s'opposèrent avec véhémence à tout rapprochement de peur d’ouvrir la voie à une
«lente réconciliation » voire à des réparations. Des campagnes furent organisées à «lente réconciliation » voire à des réparations. Des campagnes furent organisées à large échelle et il y eut de grandes manifestations dans l'ensemble du pays. Le sujet avec force contre un accord avec l'Allemagne, était dirigée par Menahem Begin et son parti Herout (Liberté). Elle accusait Ben Gourion de vouloir vendre lâme des personnes assassinées pour obtenir de l'argent allemand. « Nous ne vendrons pas le sang de nos frères et sceurs pour de l'argent! ! ou «Le sang de tes frères et seeurs crie du fin fond de la terre ! (Cenesis $4: 10-12)$ fut souvent scandé à l'époque. La

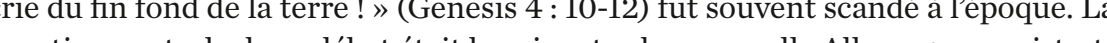
question centrale de ce débat était la suivante : la « nouvelle Allemagne » existe-t-
elle vraiment ?10

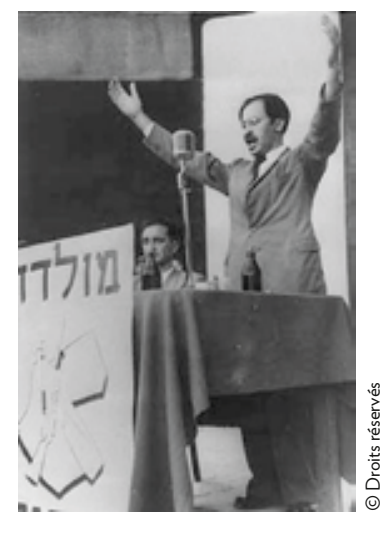

Menahem Begin début ouût 1948, quand il a fondé le parti Herout

\section{(7) À propos de la législation et}

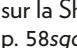

(8) Elle fut révisée et complétée

(9) À propos de la création
de Yad Vashem voir Boaz Cohen, «S Setting the Agenda
of Holocaust Research: Discord of Holocaust Research: Discord
at Yad Vashem in the 1950s $s$, in
David Bankier and Dan Michma David Bankier and Dan Michman
(ed.), Holocaust Historiography in (ed.), Holocaust Historiography in Polemics \& Achievements, (10) À propos des divergences Segev, 2003. 
a vie entière de Menahem Begin fut marquée par les souvenirs d'un judaïsme polonais florissant qui avait été anéanti en même temps que sa famille (Begin 1954, p. 249-252) . Aussi n’a-t-il pas thématisé la Shoah par simple opportunisme politique, toute sa personnalité était liée à l'expérience traumatisante de la destruction des Juifs d’Europe. Ces événements thématisés très tôt indiquent que la Shoah était devenue un composant important de l'identité israélienne et qu’elle avait une influence considérable sur la vie publique et sur le fonctionnement de la politique israélienne.

Dans les années 1950, quelques procès eurent lieu contre d’anciens Kapos juifs. La plupart des accusés avaient été reconnus par hasard dans la rue par leurs anciennes victimes. Ces procès furent cependant de courte durée : la société israélienne n’était pas encore prête à opérer une approche différenciée du passé (Itamar 2015, p. 23-40, 529-543).

La querelle sur les chapitres sombres et complexes que représentaient les Kapos voire les collaborateurs fut rapidement close. Il était préférable de se consacre aux questions principales et non à celles considérées alors comme accessoires. On voulait se souvenir des personnes assassinées, aider les survivants à commence un nouveau chapitre de leur vie et construire une patrie juive en Israël.

De manière générale, on peut constater que jusqu'en 1961, la population israélienne n’a pas toujours été très patiente dans la manière de considérer les survivants. Elle n’avait pas une « oreille compatissante » face aux récits personnels des rescapés. Il serait faux toutefois de prétendre que pendant les dix à quinze premières années suivant leur immigration, ils n'ont jamais eu la possibilité de relater leur terrible parcours. Il y a lieu de contester l'historiographie lorsqu'elle considère cette époque comme «le temps du grand silence». Les événements qui se sont produits durant la Shoah furent racontés de temps à autre, non pas sous forme de récit autobiographique, mais plutôtà la façon d’un rapport distant et «neutre». Aborder la Shoah revenait alors à calquer le récit sur la fiction, mais elle était de 作 toute façon présente. On navait pas encore atteint le stade dun échange intime. les récits demeurant anonymes et insaisissables durant les années qui suivirent. Ce n'est quau moment sonnels des survivants que la Shoah put être abordée dans sa dimension globale.

Mais comment expliquer qu’une mémoire collective puisse rester si abstraite jusque dans les annees 1950 ?

D'une part, la longue et sanglante guerre israélo-arabe et la construction d'un nouvel État monopolisèrent totalement les énergies spirituelles des « anciens » et des «nouveaux » Israéliens, ne laissant que peu de place à une réflexion sur d'autres perspectives et surl'histore sicomplexe et douloureuse de la Sho ho D'autre part, une cultur fallait au contraire cacher son deuil personnel. La devise très répandue était
«Serrer les dents et faire avancer la construction. » Lindividu en deuil trouvait tout au plus un réconfort dans le fait qu'il n'était pas le seul à partager ce destin. L'inhibition du deuil ne concernait pas seulement les victimes de la Shoah, mais aussi celles de la guerre israélo-arabe. Se tourner vers l'avenir semblait être la réaction la plus opportune et la meilleure réponse à la perte de proches et à cette douloureuse période, mais cela empêchait toute discussion approfondie sur la souffrance refoulée.

Pour les survivants, intégration et assimilation constituaient alors les objectifs principaux $^{11}$. Ils étaient à la recherche d'un travail et d'un logement, voulaient terminer leur formation ou leurs études, voire fonder une famille. La volonté de vivre et le désir de panser les plaies étaient très forts. Le refoulement venait des deux côtés. Le renouveau, la survie étaient le plus important pour les deux parties de la société. Il ne faut pas oublier que les survivants, physiquement affaiblis, désorientés et ayant subi des sévices et des humiliations, avaient souvent honte de raconter leurs expériences. Ils taisaient leur vécu même devant leurs enfants.

L’année 1961 marqua un changement radical dans le comportement de la société srálienne vis-à-vis de la Shob Cette anné israklie procès d'Adolf Eichmann, larchitecte de la «Solution finale de la question juive ». Les répercussions de ce procès furent énormes, grâce aux médias, il fut suivi par un large public, et le sujet de la shoah gagna encore en interêt. De nombreux Israéliens entendirent pour la première fois les récits personnels des souffrances des victimes. Un journaliste réputé écrivit à cette époque :

Ne connaissions-nous pas déjà toutes ces choses-là ? Oui, nous les connaissions. Même avant le procès Eichmann, nous savions déjà [...]. Mais lorsque ces documents se retrouvèrent sur la table des plaignants et qưune partie de l'acte d'accusation fut extraite du silence des archives, ce fut soudain comme si ces documents s'exprimaient pour la première fois, etce que nous avons apprisétait distinct de ce dort nous avions contaisene avat (Haim Gour cité dins Yablonka, 2000, p. 180-181).

En parallèle, une discussion s'engagea sur la façon dont on s'était comporté es années précédentes avec les survivants. Nathan Alterman, poète israélien, résuma ce changement

Aurions-nous pu accorder davantage d’attention à ces gens ? Peu importe, cette phase d'apprentissage a ouvert une ère nouvelle sur la manière dont nous abordons le passé. À l’avenir, plus aucune critique à l'égard des Juifs ayant souffert sous le régime nazi en Europe ne sera tolérée (Alterman cité dans Yablonka, 2000, p. 181-182).

Le procès fut retransmis en direct à la radio. Les gens interrompaient leur travail pour écouter les larmes aux yeux les témoignages des victimes. Le procès 
Eichmann a marqué le passage à lâge adulte de toute une génération. Quand il se termina en 1963, on constata un renforcement de la prise de conscience pour la Shoah et l'image des survivants au sein de la société israélienne s'est améliorée.

Le procès Eichmann influença aussi le traitement scientifique et institutionnel de la Shoah: Yad Vashem prit de l'importance du jour au lendemain. Avant le procès, Yad Vashem n'était encore guère significatif et peu connu. Le prestige de la recherche liée à la Shoah augmenta ausi de manière considérable et on l'enseigna alors dans lièe à la Shoah aumenta aussigna alors dans les universites. Toute une génération de jeunes historiens israéliens commença à s'y consacrer exclusivement. En historiographie, la transition ne fut pas uniquement quantitative mais aussi qualitative. On commença enfin à reconnâ̂tre la dignité humaine indépendamment du fait que l'on ait combattu les armes à la main ou non dans un contexte où l'on avait été persécuté sans pitié. Cette forme de reconnaissance fit naitre l'expression de « résilience juive $»^{12}$.

Après de nombreuses années d'intégration réussie dans la société israélienne, les survivants ont rappelé publiquement à quel point ils furent un temps rejetés en Israël. Ils expliquèrent avec assurance pourquoi Israël n’a pu compenser ce qu'ils avaient perdu, et ils provoquèrent de la sorte un débat public sur la manière dont ils avaient été traités. À partir de là, la mémoire de la Shoah devint un kaléidoscope de souvenirs personnels et rendit sa juste valeur au vécu très différent de ces per-

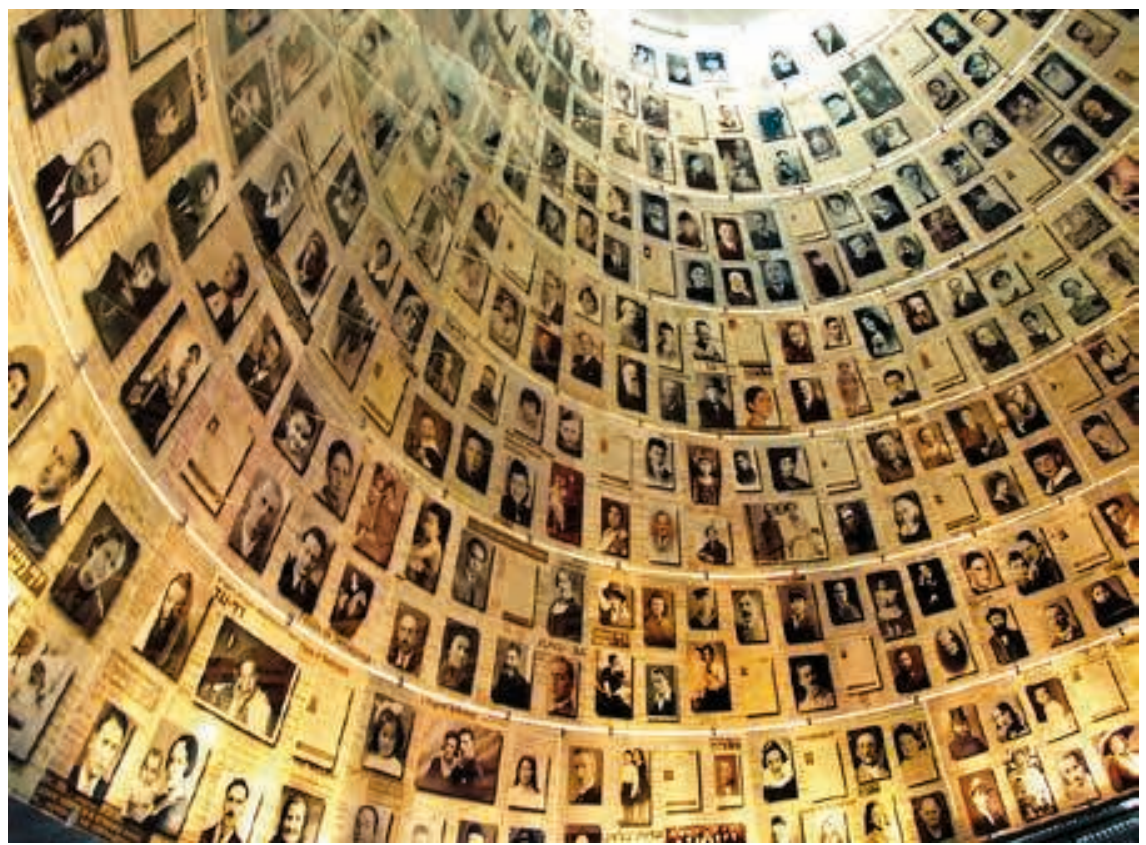

sonnes. La revendication des survivants de ne pas être perçus comme Israéliens moyens ou idéaux ; mais plutôt comme individus fut de mieux en mieux acceptée (voir Porat, 2008b, 356sqq)

\section{LA PRATIQUE DE LA MÉMOIRE EN ISRAEL AUJOURD'HU}

Depuis des décennies jusqu’à nos jours, la Shoah est une des raisons principales de la légitimité de l'État d'Israël sur le plan de la mémoire officielle. Yad Vashem est ainsi une partie incontournable du programme de visite des représentants des gouvernements étrangers ${ }^{13}$. Le mémorial officiel reste de la sorte au centre de l'attention vernements étrangers ${ }^{13}$. Le mémorial officiel reste de la sorte

Le but de la pratique mémorielle est la recherche d'une identité commune juive Le but de la pr.
tisraélienne.

Parmi les aspects importants de la culture mémorielle figurent aussi les voyages de jeunes Israéliens sur les sites de « la Solution finale de la question juive » en Pologne ; ces visites représentent désormais un facteur central de la société israélienne. Les voyages scolaires des élèves en Europe de l'Est est le signe que les jeunes montrent un grand intérêt pour la Shoah .98\% des lycées etécoles professionnelles en Israël organisent de tels voyages ${ }^{14}$. Presque chaque écolier israélien y a participé au moins une fois.

Avec l'activité du Centre Simon-Wiesenthal à Jérusalem, un élément important trop longtemps négligé a été redécouvert au sein de la société israélienne : la poursuite pénale des criminels nazis. La dernière campagne la plus récente, Operation: last chance ${ }^{15}$, consiste à trouver des criminels nazis dans les pays dans lesquels il n'était jusqu’à présent pas évident de les traîner en justice, comme la Lituanie, la Lettonie, l'Ukraine ou la Pologne. Avec l'annonce de récompenses pour des informations obtenues de la population et aboutissantà l'inculpation d'un criminel nazi, cette action a connu un grand sucès ces dernières années et a permis de profiter le lemière ocearion de les condanner par voie de justice.

Les récits personnels des survivants occupent une place toujours plus importante dans le débat de société autour de la Shoah. Des survivants se sont donné pour mission de visiter des écoles et des casernes afin de partager leur vécu pour que les jeunes generations apprennentà mieuxcontre le sujet. Lors de la visite des sites de crimes, des survivants accompagnent souvent les groupes de jeunes gens. Sur place, leur récit est d'autant plus authentique. Aujourd'hui, il est remarquable de constater que les rescapés disposés à s’exprimer sur leur douloureux passé sont plus nombreux qu'il y a encore vingt ans. Cette tendance est illustrée par la publication de nombreux livres par des survivants et sexplique par un chan lo pution de nomre le lattitude de la société a légard de la Shoah et des rescapes. Les conditions dans
(13) C'est ainsi que dans un délai rapproché en avil 1997 , tant le
ministre allemand des Affaires étrangères, Sigmar Gabriel, que Ple rens to Poland (14) Pupil's Delegations to Poland
Submitted to the Knesset's Committee of Elucation, Culture
and Sport, 29 janvier 2008 . (15) Pour plus dinformations, voir
le site de la campagne : http:/// www.operationlastchance.org 
dernières décennies, ce qui a ouvert de nouvelles opportunités dans la manière d’aborder le passé

Mais la transmission personnelle des souvenirs ne sera plus longtemps possible du fait du temps qui passe. Passée leur retenue initiale, les survivants souhaitent à présent léguer leur récit de la Shoah avant qu'il ne soit trop tard. De nombreux sites mémoriaux dans le monde ont reconnu l'urgence de la situation et s'efforcent de recueillir les témoigna des derniers rescapés par écrit, par le son et par l'image recueil res lén ex vidéo ${ }^{16}$.

Pour des raisons de facilité, les interviews sont souvent réalisées au domicile des survivants, qui ont pris de lâge. Des entretiens avec des témoins lors de manifestations dans les mémoriaux sont aussi enregistrés.

La Shoah joue également un rôle important au sein de l'opinion publique israélienne dans le cadre des relations israéliennes avec la Pologne.

Les publications de l'auteur judéo-polono-américain Jan Tomasz Gross n’ont guère surpris nombre d'Israéliens. Dans son fameux livre Neighbours ${ }^{17}$ Gross a monguère surpris nombre dsraéliens. Dans son fameux livre Neighbours Gross a montré que dans certaines localités, ce n'etaient pas les Allemands, mais leurs voisin. polonais qui furent responsables de nombreux assassinats de Juifs. À Jedwabne, 1600 Juifs ont été brûlés vifs dans une grange par des villageois polonais. En Pologne, la publication de ce livre a suscité des réflexes de défense, tandis qu'en Israël, les découvertes de Gross n’ont étonné pratiquement personne.

Gross a récemment publié un autre livre qui évoque aussi un pogrom à Kielce, au cours duquel plus de 40 survivants de la Shoah ont été assassinés en $1946^{18}$.

L’image de la Pologne en Israël s'est fortement dégradée du fait des livres de Gross. La situation politique entre les deux pays s'est d'autre part énormément Gross. apaisée. La Pologne est devenue l'un des partenaires les plus proches d'Israël et l'a toujours soutenu sur le plan international au cours de la dernière décennie. La presse polonaise présente Israël presque toujours de manière positive. Dans ce processus de rapprochement, il n'y eut seulement une courte parenthèse sous la présidence du Premier ministre Jarosław Aleksander Kaczyński, au pouvoir de 2006 à 2007.

Celui-ci avait formé une coalition avec deux partis particulièrement antijuifs, si ce n’est antisémites, dont le Parti pour la famille. De plus, les émissions de la station de radio polonaise antisémite Radio Maryja et la nomination du ministre de l'Éducation Maciej Giertych, qui s'était prononcé contre les voyages de la jeunesse israélienne en Pologne ont perturbéles relations.Celles ci se sont nettementaméliorées par après ${ }^{19}$. Les développements plus récents suite au nouveau changement de gouvernement en Pologne restent à observer.
La discussion sur le rôle de la Pologne pendant la Shoah a montré que le débat sur des sujets plus complexes en rapport avec celle-ci est devenu moins problématique. La perception de la réalité historique n'est plus à sens unique, comme ce fut le cas au départ, des thèmes à double tranchant et d'une plus grande complexité peuvent faire l'objet d'un débat différencié, mené sur une large échelle.

Un autre événement récent qui a soulevé l'indignation de la société israélienne fut le fait d'apprendre que plus de la moitié des survivants de la Shoah vivait en

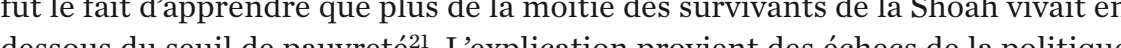
dessous du seuil de pauvreté2. Liexplication provient des échecs de la politique gouvernementale pendant des décennies et de la corruption sévissant dans des organisationdestation de l'opinion publique a poussé le gouvernement à annoncer son intention d'aider les survivants avant qu'ils ne disparaissent, mais ce n'était pas assez et elle est intervenue trop tard. Jusqu’à aujourd'hui, pas un shekel n'a encore été versé. Des voix critiques craignent que le gouvernement temporise jusqu’à ce que les survivants décèdent. L'évidence selon laquelle les survivants de la Shoah ont un statut respecté au sein de la société israélienne est donc malheureusement illusoire et nous remarquons

Des statisticiens ont calculé que chaque jour, 35 à 60 survivants de la Shoah décèdent ${ }^{21}$. Devant cette perte inéluctable, existe toutefois un aspect positif: les deuxième et troisième génération des enfants nés après la Shoah s'expriment davantage sur ce qu'ils éprouvent par rapport à ce passé. Ils sont nombreux à publier des biographies et à participer aux Johrzeiten, ces commémorations au cours desquelles les communes se souviennent de leurs victimes. Ils ont commencé eux-mêmes à raconter aux gens intéressés les histoires de leurs parents, comme si c'était leur propre histoire. Ils mesurent de plus en plus l'importance qu'a l'histoire de leurs parents et grands-parents pour eux-mêmes. Ces générations se sentent souvent plus concernées par les expériences de leurs parents que ce qu’on soupçonneraitau premier abord (Wardi, 1997). En s'identifiant à leurs familes ils se sonten se la Shoah, nais drue manière bien évidemment complètement diférente de celle de leurs parentset gands-parents qui avro vécu eux-mêmes ces atrocités.

Lorsqu'on lit la presse israélienne, on remarque quelque chose hors du commun qui ne fait que confirmer limpression relatée ci-dessus : il he se passe pas un jour sans que paraisse une information qui a un lien direct ou indirect avec la Shoah. Si elle fait partie de l'histoire israélienne, elle appartient aussi au présent israélien sous la forme d’information traitée au jour le jour dans les médias. La discussion concernant la Shoah ne sera pas clôturée de sitôt.

Traduit de lallemand par Anna Gorbatova. Révisé par le secrétariat de rédaction.
(20) Voir Gisela Dachs, Nach KZ Zeit, 24 avril 2008 ; htt zeit.de/2007/43/Nach_KZ_undVertreibung_die, consulté le 10
mai 2017 .

(21) Voir 30 Holocaust Survivors
Die Every Day in Israel, in Maariv es mai 2011 et 37 Holocaust Survivors Die D Diliy in lsrael, in
Globs, 3 avril 2013. 
Les étapes de la discussion sur la Shoah dans la société israélienne (suite)

\section{BIBLIOGRAPHIE}

- Yehuda Bauer, Jewish Reactions to the Holocaust, Tel Aviv, 1989, p. 217sqq.

- Menachem Begin, «Three Things », in Encyclopedia of Diaspora, Brisk de-Litta, Tel Aviv, 1954, p. 249 -252.

- Boaz Cohen, «Holocaust Heroics. Ghetto Fighters and Partisans in Israeli Society and Historiography », in Journal of Political and Military Sociology, volume 31, $\mathrm{n}^{\circ}$ 2, 2003, p. 197-203.

- ---, «Setting the Agenda of Holocaust Research: Discord at Yad Vashem in the 1950s », in David Bankier and Dan Michman (dir.), Holocaust Historiography in Context. Emergences Challenges Polemics \& Achievements, Jérusalem, 2008, p. 255-292.

- Gisela Dachs, Nach KZ und Vertreibung die Armut, in Die Zeit, 24 avril 2008, version online : http://www.zeit. de/2007/43/Nach_KZ_und_Vertreibung_die, consulté le 10 mai 2017.

- Jan T. Gross, Neighbors. The Destruction of the Jewish Community in Jedwabne, Poland, Princeton University Press, 2001.

- ---, Fear: Anti-Semitism in Poland After Auschwitz, Princeton University Press, 2006.

- Levin Itamar, Kapo in Allenby, Jerusalem, Yad Ben Zvi \& Moreshet Publications, 2015, p. 23-40, 529-543.

- Dan Michman, Pour une historiographie de la Shoah. Conceptualisations, terminologie, définitions et problèmes fondamentaux, Paris, In Press, 2001.

- Jan Opielka, Beständig gut, in Jüdische Allgemeine, $1^{\text {er }}$ novembre 2007, version online : http://www.juedischeallgemeine.de/article/view/id/4827, consulté le 10 mai 2017.

- Dina Porat, «First Testimonies on the Holocaust: The Problematic Nature of Conveying and Absorbing them, and the Reaction in the Yishuv ", in David Bankier and Dan Michman (dir.), Holocaust Historiography in Context. Emergences Challenges Polemics \& Achievements, Jerusalem, Yad Vashem, and New York: Berghahn Books, 2008, p. 437-460.

- Dina Porat, Israeli Society, the Holocaust and its Survivors, London, Vallentine Mitchell, 2008b, p.11sqq.

- --- Sefer Hapartizanim Hayehudim, Tel Aviv, 1959.

- --- Sefer Hashomer Hatzair, volumes 1-3, Merhavia', 1956.

- --- Sefer Milhemet Hagetaot, Tel Aviv, 1953.

- Tom Segev, Le septième million. Les Israéliens et le génocide, Paris, Liana Levi, 2003.

- s.n., 30 Holocaust Survivors Die Every Day in Israel, Maariv, $1^{\text {er }}$ mai 2011.

- s.n., 37 Holocaust Survivors Die Daily in Israel, Globs, 3 avril 2013.

- Dina Wardi, Siegel der Erinnerung. Das Trauma des Holocaust - Psychotherapie mit den Kindern der Überlebenden, Stuttgart, Klett-Cotta, 1997.

- Yechiam Weitz, "The Holocaust on Trial. The Impact of the Kasztner and Eichmann Trials on Israeli Society », Israel Studies 1 (2), 1996, p. 1-26.

- Hanna Yablonka, «The Silent Partner. Holocaust Survivors in the IDF », in Selwyn Ilian Troen \& Noah Lucas (dir.), Israel: The First Decade of Independence, State University of New York Press, 1995, p. 557-572.

- ---, Survivors of the Holocaust: Israel after the War, Londres, 1999, p. 82.

- ---, The State of Israel against Adolf Eichmann, Tel Aviv 2000, p. 180-181.

- Idith Zertal, Israel's Holocaust and the Politics of Nationhood, Cambridge University Press, 2005, p. 58sqq. 